\title{
Primeiro registro da ocorrência de Cirrospilus floridensis Evans (Hymenoptera) como parasitóide de Phyllocnistis citrella Stainton (Lepidoptera) no Brasil
}

\author{
First record of occurrence of Cirrospilus floridensis Evans (Hymenoptera) as a parasitoid of \\ Phyllocnistis citrella Stainton (Lepidoptera) in Brazil
}

Simone Mundstock Jahnke ${ }^{1}$ Luiza Rodrigues Redaelli² Lúcia Maria Guedes Diefenbach $^{3}$

\section{- NOTA -}

RESUMO

Este estudo relata o primeiro registro de Cirrospilus floridensis Evans no Brasil. Este Eulophidae foi obtido de pupas de Phyllocnistis citrella Stainton (minadordas-folhas-dos-citros) presentes em folhas de bergamoteiras da variedade Montenegrina (Citrus deliciosa) e do híbrido tangor Murcott (C. sinesnis $x \boldsymbol{C}$. reticulata) coletadas de julho de 2001 a junho de 2003 em Montenegro, RS, Brasil. Foram registrados adultos, machos e fêmeas de $\boldsymbol{C}$. floridensis.

Palavras-chave: Insecta, Eulophidae, Gracillariidae, citros, ectoparasitóide, minador-dos-citros.

\section{ABSTRACT}

This study reports the first record of Cirrospilus floridensis Evans in Brazil. This Eulophidae was obtained from pupae of Phyllocnistis citrella Stainton (citrus-leafminer) present in infested leaves of the Montenegrina variety (Citrus deliciosa) and the hybrid tangor Murcott (C. sinensis $x \boldsymbol{C}$. reticulata) collected from July of 2001 to June of 2003 in Montenegro, RS, Brazil. Adults, males and females of $\boldsymbol{C}$. floridensis were registered.

Key words: Insecta, Eulophidae, Gracillariidae, citrus, ectoparasitoids, citrus-leafminer.

\section{Phyllocnistis citrella Stainton} (Lepidoptera: Gracillariidae) é nativa do sudeste asiático, estando atualmente dispersa por países produtores da Oceania, África, Europa e Américas (HEPPNER, 1993). No Brasil, foi detectada pela primeira vez em 1996, no estado de São Paulo (GRAVENA, 1996), sendo que, em 1997, grande parte dos estados produtores de citros já registrava sua presença.

Associado ao minador-das-folhas-decitros encontra-se um grande número de inimigos naturais, especialmente parasitóides. Dentre estes, os da família Eulophidae têm merecido destaque, com cerca de 24 espécies (CÔNSOLI et al., 1996). No Brasil, foram catalogados os eulofídeos pertencentes aos gêneros Sympiesis, Quadrasticus, Cirrospilus e Galeopsomyia. No estado de São Paulo, Galeopsomyia fausta LaSalle é a espécie mais freqüente entre os parasitóides de $\boldsymbol{P}$. citrella (COSTA et al., 1999).

O gênero Cirrospilus tem distribuição conhecida para as regiões tropicais e subtropicais do Novo Mundo, sendo que a maioria das espécies são parasitóides do minador. Este trabalho visou ao registro de uma espécie desse gênero como parasitóide de pupas de $\boldsymbol{P}$. citrella em bergamoteiras em Montenegro, RS.

A coleta dos parasitóides foi realizada no município de Montenegro ( $29^{\circ} 68^{\prime} \mathrm{S}$ e $\left.51^{\circ} 46^{\prime} \mathrm{O}\right)$ em dois pomares contíguos de bergamotas, variedade

${ }^{1}$ Biólogo, MSc em Fitotecnia, Departamento de Fitossanidade, Universidade Federal do Rio Grande do Sul (UFRGS), Av. Bento Gonçalves, 7712, 91540-000, Porto Alegre, RS, Brasil. E-mail:smjahnke@yahoo.com. Autor para correspondência. ${ }^{2}$ Engenheiro Agrônomo Professor, Doutor, Departamento de Fitossanidade, UFRGS.

${ }^{3}$ Biólogo, Doutor, Instituto de Pesquisa Biológica (IPB), Laboratório Central do Estado (LACEN)/RS, Fundação Estadual de produção e Pesquisa em Saúde (FEPPS), Av. Ipiranga, 5400, 90610-000, Porto Alegre, RS, Brasil. 
Montenegrina (Citrus deliciosa) e híbrido Murcott (C. sinesnis $x \boldsymbol{C}$. reticulata), com aproximadamente um ha cada um, totalizando 312 plantas.

No primeiro ano, a cada ocasião de amostragem, foram sorteadas aleatoriamente 12 plantas (aproximadamente 4\% das árvores) de cada pomar, de onde foram retiradas as unidades de amostra que consistiam de folhas contendo pupas de $\boldsymbol{P}$. citrella. As amostragens foram realizadas quinzenalmente de julho de 2001 a junho de 2002. No segundo ano de amostragem (julho de 2002 a junho de 2003), buscando aumentar o número de indivíduos amostrados, optouse por monitorar 24 plantas de cada pomar. O número de unidades amostrais foi estimado com base no resultado obtido no teste de suficiência amostral (ELLIOT, 1983).

Em cada planta sorteada, foram inspecionados todos os brotos para verificar a presença de pupas de $\boldsymbol{P}$. citrella. Todas as folhas contendo pupas foram coletadas. Em laboratório, as folhas foram individualizadas em placas de Petri, vedadas e mantidas, no laboratório, em condições naturais de temperatura e umidade, até a emergência dos parasitóides ou dos adultos de $\boldsymbol{P}$. citrella.

A identificação dos parasitóides, ao nível de família foi realizada através de chave dicotômica
(PENTEADO-DIAS et al., 1997). Para identificação genérica, os exemplares foram enviados ao Dr. Valmir Antônio Costa do Instituto Biológico de Campinas. A identificação específica foi realizada pelo Dr. John La Salle, do CSIRO Entomology, da Austrália.

Dentre os parasitóides amostrados foi identificada a presença de Cirrospilus floridensis Evans (Hymenoptera: Eulophidae) espécie descrita por Evans em 1999 e conhecida por desenvolver-se como ectoparasitóide de $\boldsymbol{P}$. citrella (EVANS, 1999). Outras espécies desse gênero, tais como $\boldsymbol{C}$. ingenuus e $\boldsymbol{C}$. quadristriatus parasitam seus hospedeiros nas fases de prepupa e $\boldsymbol{C}$. phyllocnistoides no segundo e terceiro ínstar larval (URBANEJA et al., 1998; URBANEJA et al., 2000). Entretanto, o conhecimento a respeito da biologia de C. floridensis é escasso, uma vez que só recentemente essa espécie foi descrita.

C.floridensis apresenta coloração geral amarelada ou alaranjada, com algumas áreas marrom-escuras ou verde-metálico-escuras no tórax. Os adultos têm em torno de $1,7 \mathrm{~mm}$ de comprimento (SHAUFF et al., 1998). Segundo EVANS (1999), esta espécie pode ser diferenciada de outras do mesmo gênero pela faixa escura transversal ao longo da margem posterior do lobo torácico médio.

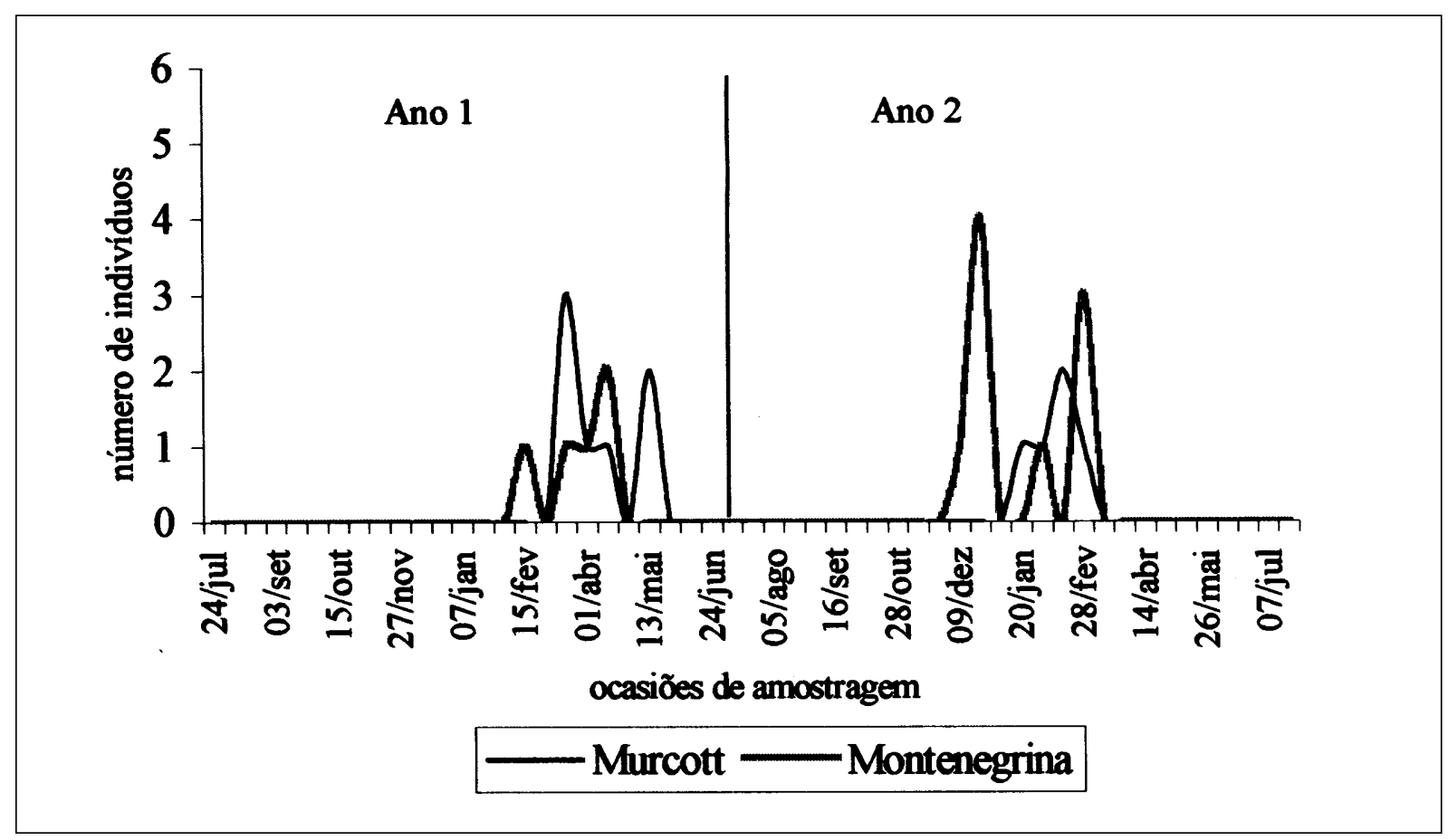

Figura 1 - Flutuação da população de Cirrospilus floridensis em pomares de bergamoteiras (variedade Montenegrina e híbrido tangor Murcott) em Montenegro, RS. Ano 1: julho de 2001 a junho de 2002; Ano 2: julho de 2002 a junho de 2003.

Ciência Rural, v.35, n.2, mar-abr, 2005. 
O primeiro espécime de $\boldsymbol{C}$. floridensis emergiu de uma pupa de $\boldsymbol{P}$. citrella coletada em 15 de fevereiro de 2002 na variedade Montenegrina. Em Murcott, a presença foi detectada em 18 de março de 2002. Nos dois anos de amostragem, foram registrados 26 indivíduos adultos (11 machos e 15 fêmeas) de $\boldsymbol{C}$. floridensis emergidos de pupas coletadas nos pomares de citros.

No primeiro ano de amostragem, a espécie esteve presente de fevereiro a maio de 2002. No segundo ano, detectou-se a sua presença de dezembro de 2002 a fevereiro de 2003 (Figura 1). Cabe ressaltar que a espécie hospedeira, $\boldsymbol{P}$. citrella, foi registrada na área de setembro a junho no primeiro ano e de outubro a março, no segundo. Esta variação pode ser devida à alternância de produção de frutos e brotos, característica de tangores e tangerinas, nas quais, em um ano, há excessiva produção de frutos e no ano seguinte, maior emissão de brotações vegetativas (DOMINGUES et al., 2001). Um outro aspecto que pode ter interferido na flutuação populacional de $\boldsymbol{C}$. floridensis são as variações das condições climáticas entre os dois anos (RODRIGUES $\&$ DORNELLES, 1999) uma vez que seu hospedeiro também sofreu modificação na população devido a alterações nas condições gerais de temperatura e umidade (JESUS et al., 2004).

A presença de $\boldsymbol{C}$. floridensis já é conhecida da Flórida até a Venezuela sendo que o presente trabalho constitui-se no primeiro registro da sua ocorrência no Brasil.

\section{AGRADECIMENTOS}

Ao Dr. Valmir Antônio Costa do Instituto Biológico de Campinas pela identificação genérica e ao Dr. John La Salle CSIRO Entomology (Austrália), pela identificação da espécie. À CAPES e ao CNPq pela concessão das bolsas de pesquisa ao primeiro e segundo autor, respectivamente.

\section{REFERÊNCIAS BIBLIOGRÁFICAS}

COSTA, V.A. et al. Indigenous parasitoids (Hy., Chalcidoidea) of Phyllocnistis citrellaStainton (Lep., Gracillariidae) in Jaguariúna, São Paulo State, Brazil: preliminary results. Journal of Applied Entomology, Hamburg, v.123, p.237-240, 1999
CÔNSOLI, F.L. et al. Phyllocnistis citrella Station,1856 (Lepidoptera: Gracillariidae: Phyllocnistinae) - A Lagarta minadora dos citros. Piracicaba : FEALQ, 1996. 39 p.

DOMINGUES, M.C.S. et al. Reguladores vegetais e o desbaste químico de frutos de tangor murcose. Scientia Agricola Piracicaba, v.58, n.3, p.487-490, 2001.

ELLIOT, J.M. Some methods for the statistical analysis of samples of benthic invertebrates. Ambleside : Freshwater Biological Association, 1983. 156p.

EVANS, G.A. A new species of Cirrospilus (Hymenoptera: Eulophidae) and two synonymes of parasitoids reared from the citrus leafminer, Phyllocnistis citrella (Lepidoptera: Gracillariidae). Florida Entomologist, Gainesville, v.82 n.3, p.448-453, 1999.

GRAVENA, S. Lagarta minadora dos citros no Brasil. Laranja, Cordeirópolis, v.17, n.1, p.286-288, 1996.

HEPPNER, J.B. Citrus leafminer, Phyllocnistis citrella, in Florida (Lepidoptera: Gracillariidae: Phyllocnistinae). Tropical Lepidoptera, Gainesville, v.4, p.49-64, 1993.

JESUS, C.R. et al. Mortalidade das larvas de Phyllocnistis citrella Stainton, 1856 (Lepidoptera: Gracillariidae) por predação em pomares de tangerineira em Montenegro, RS - Brasil. In: SIMPÓSIO DE CONTROLE BIOLÓGICO, 8., 2003, São Pedro, SP. Resumos... Piracicaba, SP : Sociedade Entomológica do Brasil, 2003. 206p. p.149.

PENTEADO-DIAS A.M. et al. Parasitóides de Phyllocnistis citrella Stainton (Lep. Gracillariidae: Phyllocnistinae) no estado de São Paulo. Laranja, Cordeirópolis, v.18, n.1, p.79-84, 1997.

RODRIGUES, L.R.; DORNELLES, A.L.C. Origem e caracterização horticultural de tangerina "Montenegrina". Laranja, Cordeirópolis, v.20, n.1, p.153-166, 1999 .

SCHAUFF, M.E. et al. The Geneara of Chalcid parasitoids (Hymenoptera: Chalcidoidea) of citrus leafminer Phyllocnistis citrella Stainton (Lepidoptera: Gracillariidae). Journal of Natural History, London, v.32, p.1001-1056, 1999

URBANEJA, A. et al. Dinâmica e impacto de los parasitoides autocotnos de Phyllconistis citrella Stainton, en la comunidad Valenciana. Investigacion Agraria: Producción Vegetal, Valência, v.13, n.3, p.409-423, 1998.

URBANEJA, A. et al. Indigenous natural enemies associated with Phyllocnistis citrella (Lepidoptera: Gracillariidae) in Eastern Spain. Biological Control, Orlando, v.18, n.3, p.199-207, 2000 